\title{
PRODUCCIÓN CIENTÍFICA DE LA UNIÓN EUROPEA EN EL CAMPO DEL TURISMO (1998-2017)
}

\author{
José RAMON CARDONA \\ E. U. de Turismo del C.I.E., Universitat de les Illes Balears, España \\ josramcardona@gmail.com \\ María Dolores SÁNCHEZ-FERNÁNDEZ \\ Facultad de Economía e Empresa, Universidade da Coruña, España \\ msanchezf@udc.es
}

\begin{abstract}
Resumen
Europa ha liderado el turismo internacional desde sus inicios pero en el campo de la investigación turística no está tan claro. Medir los resultados de la actividad investigadora es difícil, pero el volumen de artículos en revistas internacionales es la aproximación más habitual. El objetivo de este trabajo es realizar un análisis bibliométrico para determinar el estado de la investigación turística en la Unión Europea. Para ello se tomó en consideración artículos en revistas académicas especializadas, publicados entre 1998 y 2017 e indexados en Scopus. En el análisis destacan dos tipos de países por su producción científica: los países del Mediterráneo, excepto Italia y Francia, y países con alta implicación en investigación (Reino Unido, Finlandia, Dinamarca, Suecia y Austria). Entre los países con producción baja, hay dos grupos: diversos países con poca especialización en turismo (Luxemburgo, Alemania, Francia, Bélgica e Italia) y países del este (Lituania, Polonia, Letonia, Republica Checa, Rumanía, Hungría, etc.). La falta de tradición en investigación turística de algunos países, el bajo nivel económico de los países del este y el sesgo lingüístico de las publicaciones, que beneficia a los países anglosajones, son los principales retos que deben afrontarse.
\end{abstract}

Palabras clave: Turismo, Investigación, Unión Europea, Análisis Bibliométrico, Scopus.

\begin{abstract}
Europe has led international tourism since its inception but in the field of tourism research is not so clear. Measuring the results of research activity is difficult, but the volume of articles in international journals is the most usual approach. The objective of this work is to carry out a bibliometric analysis to determine the state of tourism research in the European Union. For this, articles in specialized academic journals, published between 1998 and 2017 and indexed in Scopus, was taken into consideration. The analysis highlights two types of countries for their scientific production: the countries of the Mediterranean, except Italy and France, and countries with high involvement in research (United Kingdom, Finland, Denmark, Sweden and Austria). Among the countries with low production, there are two groups: several countries with little specialization in tourism (Luxembourg, Germany, France, Belgium and Italy) and Eastern countries (Lithuania, Poland, Latvia, Czech Republic, Romania, Hungary, etc.). The lack of tradition in tourist research of some countries, the low economic level of Eastern countries and the linguistic bias of the publications, which benefits the Anglo-Saxon countries, are the main challenges that must be faced.
\end{abstract}

Keywords: Tourism, Research, European Union, Bibliometric Analysis, Scopus. 


\section{Introducción}

El turismo es uno de los sectores económicos más importantes y ha crecido mucho en las últimas décadas. En 2016, hubo 1.235 millones de turistas internacionales en el mundo que generaron unos ingreso de 1.220 .000 millones de dólares (UNWTO, 2017). Europa ha liderado el turismo internacional desde sus inicios y, en 2016, los 28 países de la Unión Europea (UE) recibieron 500 millones de turistas internacionales (UNWTO, 2017), lo que representa el 40,5\% del turismo mundial. Cabe indicar que la distribución del turismo internacional por países no es homogénea (Figura 1). En algunos países la presión turística es baja, como en Rumanía $(0,11)$, Alemania $(0,44)$ y Polonia $(0,45)$, pero en otros países es muy elevada, como en Austria $(3,21)$, Croacia $(3,22)$ y Malta $(4,72)$.

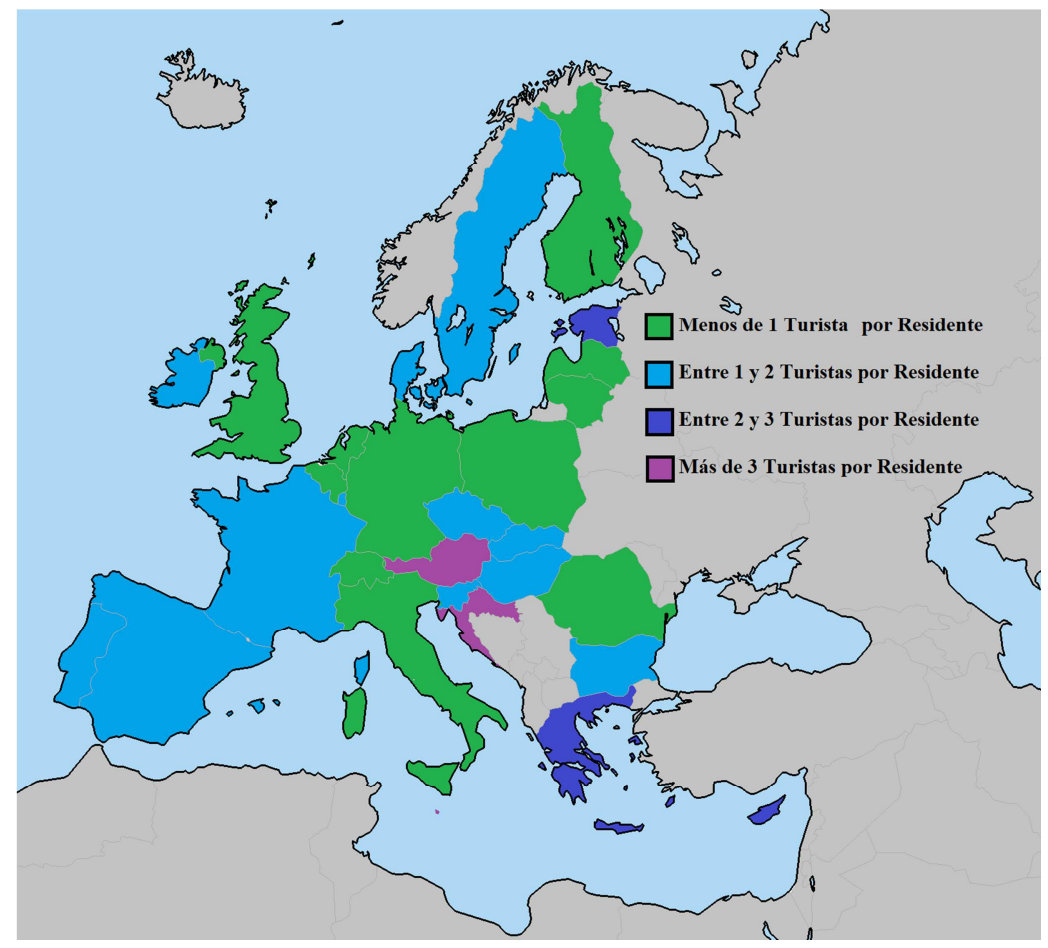

Figura 1 - Peso del turismo internacional en la UE. Fuente: UNWTO (2017) y elaboración propia.

El turismo, como todo sector económico, debe desarrollarse apoyado por una actividad investigadora que aporte nuevos conocimientos. Aunque la investigación en turismo tiene un desarrollo muy reciente (PARK, PHILLIPS, CANTER \& ABBOTT, 2011; VARGAS, 2011), en la actualidad posee un importante volumen de producción y presencia mundial (RAMÓN, 2017). La UE tiene un tradicional liderazgo en el turismo internacional (UNWTO, 2017), pero en investigación turística no está tan claro que la UE disfrute de esa misma posición.

En las últimas décadas hay una tendencia globalizadora y está ampliamente aceptado que hay que competir en un contexto mundial, más aún en el campo de la investigación que siempre ha tenido un contexto internacional. Desde los años cincuenta, Estados Unidos ha liderado la investigación en 
la mayoría de los campos, también en el turismo (SHELDON, 1991), y ello conlleva que las publicaciones en inglés se han convertido en sinónimo de ciencia internacional. Esto ha representado una ventaja estratégica para los países anglófonos y un reto añadido para el resto del mundo.

Medir los resultados de la actividad investigadora es difícil pero el volumen de artículos en revistas internacionales indexadas es la aproximación más habitual (JOGARATNAM, CHON, MCCLEARY, MENA \& YOO, 2005; LI \& XU, 2015; RYAN, 2005). El objetivo de este trabajo es realizar un análisis bibliométrico de los artículos publicados en revistas especializadas e indexadas en Scopus en los últimos veinte años, para determinar el estado de la investigación turística en la UE. También se busca determinar los centros de investigación y autores de alta producción internacional. En la actualidad la UE está compuesta por 28 países que totalizan una superficie de $4.479 .968 \mathrm{~km}^{2}$ con una población total de 516.195.432 (CENTRAL INTELLIGENCE AGENCY). El hecho de recibir 500 millones de turistas (UNWTO, 2017) implica que hay, de media, un turista por cada residente.

\section{Revisión de la Literatura}

Desde los años cincuenta el crecimiento del mundo académico ha sido exponencial, primero por el desarrollo de los países occidentales (principalmente Europa, Norteamérica, Japón, Australia y Nueva Zelanda) y más recientemente por el desarrollo de las economías emergentes (destacando China). El gran tamaño alcanzado ha obligado a la aparición de análisis que sirvan de referencia a los investigadores (JAMAL, SMITH \& WATSON, 2008; MCKERCHER, 2005; RYAN, 2005): "Whitelists" de revistas académicas; bases de datos de publicaciones; catálogos de expertos; rankings de universidades, y un largo etcétera. Todos estos análisis buscan diferenciar entre los que tienen prestigio y resultados, y los que no tienen resultados o son fraudulentos.

La investigación académica en turismo es tardía, en comparación con otros campos, pero su rápido crecimiento en las últimas décadas (PARK et al., 2011; VARGAS, 2011) ha obligado a realizar diversos tipos de análisis bibliométricos. Frecuentemente, la literatura ha buscado determinar cuáles eran las publicaciones académicas más importantes (BALLANTYNE, PACKER \& AXELSEN, 2009; CHANG \& MCALEER, 2012; FERREIRA, DEFRANCO \& RAPPOLE, 1994; FRECHTLING, 2004; HALL, 2011; KIM, SAVAGE, HOWEY \& VAN HOOF, 2009; MCKERCHER, LAW \& LAM, 2006; PECHLANER, ZEHRER, MATZLER \& ABFALTER, 2004; RYAN, 2005; SVENSSON, SVAERI \& EINARSEN, 2009; ZHAO \& RITCHIE, 2007), y las tres revistas consideradas tradicionalmente como las más relevantes en turismo son Annals of Tourism Research, Journal of Travel Research, Tourism Management (LI \& XU, 2015; MCKERCHER et al., 2006; PECHLANER et al., 2004; RYAN, 2005; SHELDON, 1991).

En otros casos se han analizado los autores (JOGARATNAM et al., 2005; LI \& XU, 2015; MCKERCHER, 2008; PARK et al., 2011; ZHAO \& RITCHIE, 2007) y las universidades (JOGARATNAM et al., 2005; LI \& XU, 2015; RYAN, 2005) con mayor producción de artículos. En los años ochenta lideraba en publicaciones University of Hawaii seguida de University of Waterloo en Canadá (SHELDON, 1991), en los noventa el liderazgo es de Texas A\&M University seguida por 
Hong Kong Polytechnic University (JOGARATNAM et al., 2005) y en el nuevo siglo Hong Kong Polytechnic University lidera a mucha distancia del resto de centros (LI \& XU, 2015; SEVERT, TESONE, BOTTORFF \& CARPENTER, 2009).

Los resultados de estos estudios son de gran utilidad para los investigadores porque aportan información sobre: las revistas científicas mejor valoradas (BALLANTYNE et al., 2009; CHANG \& MCALEER, 2012; HALL, 2011; KIM et al., 2009; LI \& XU, 2015; MCKERCHER et al., 2006; PECHLANER et al., 2004; RYAN, 2005; SVENSSON et al., 2009; ZHAO \& RITCHIE, 2007); los investigadores más productivos (JOGARATNAM et al., 2005; LI \& XU, 2015; MCKERCHER, 2008; PARK et al., 2011; RYAN, 2005; ZHAO \& RITCHIE, 2007); los centros líderes en el campo (JOGARATNAM et al., 2005; LI \& XU, 2015; RYAN, 2005; SEVERT et al., 2009). Esta información resulta fundamental para las personas que inician una carrera investigadora y necesitan planificar su futuro de la forma más exitosa posible.

Cabe indicar que también es fundamental que la información sobre los centros, autores y revistas este basado en datos objetivos, para evitar que las percepciones subjetivas induzcan a sobreestimar centros, autores y revistas que los datos objetivos muestran secundarios, en decadencia o superados. Un ejemplo son las evoluciones de University of Hawaii y Hong Kong Polytechnic University (JOGARATNAM et al., 2005; LI \& XU, 2015; SEVERT et al., 2009).

\section{Metodología}

Este trabajo es un estudio bibliométrico basado en el análisis de los artículos y revisiones indexadas en Scopus y pertenecientes a una selección de 80 revistas académicas especializadas en turismo, hospitalidad y ocio. Para el periodo 1998-2017 se encontraron 33.150 documentos que cumplían estos requisitos y, de estos documentos, 10.102 (30,5\%) tenían algún autor radicado en países de la UE. Dentro de la base de datos analizada se profundizó en los 28 países de la UE, indicando la producción total, la evolución, y los centros y autores que destacan por número de publicaciones.

\section{Resultados}

Dentro de los países con mayor producción científica (Figura 2) destacan los anglosajones (Estados Unidos, Reino Unido, Australia, Canadá y Nueva Zelanda) y los asiáticos (China, Hong Kong, Taiwán y Corea del Sur). Dentro de la UE, los mejor situados son Reino Unido (2$)$, España

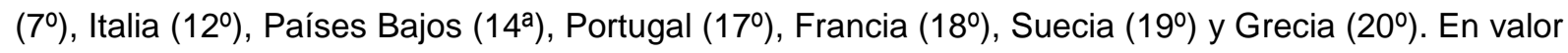
absoluto, Reino Unido (4.358 documentos) y España (1.435 documentos) superan ampliamente al resto. El Reino Unido participa en el 13,15\% de la producción mundial, España en el 4,33\% y ningún otro país europeo posee más del $2 \%$ de la producción mundial (Tabla I).

Dentro de los países analizados se han encontrado 156 centros de investigación con 20 o más publicaciones en el periodo 1998-2017. De estos centros, 6 se encuentran entre los primeros 50 del 
mundo (todos ellos en el Reino Unido), 22 entre los 100 primeros del mundo y 41 entre los 150 primeros del mundo (Tabla I). Estos centros más destacados son los líderes de la investigación en turismo, hospitalidad y ocio del continente y son los más interesantes para futuros estudiantes e investigadores.

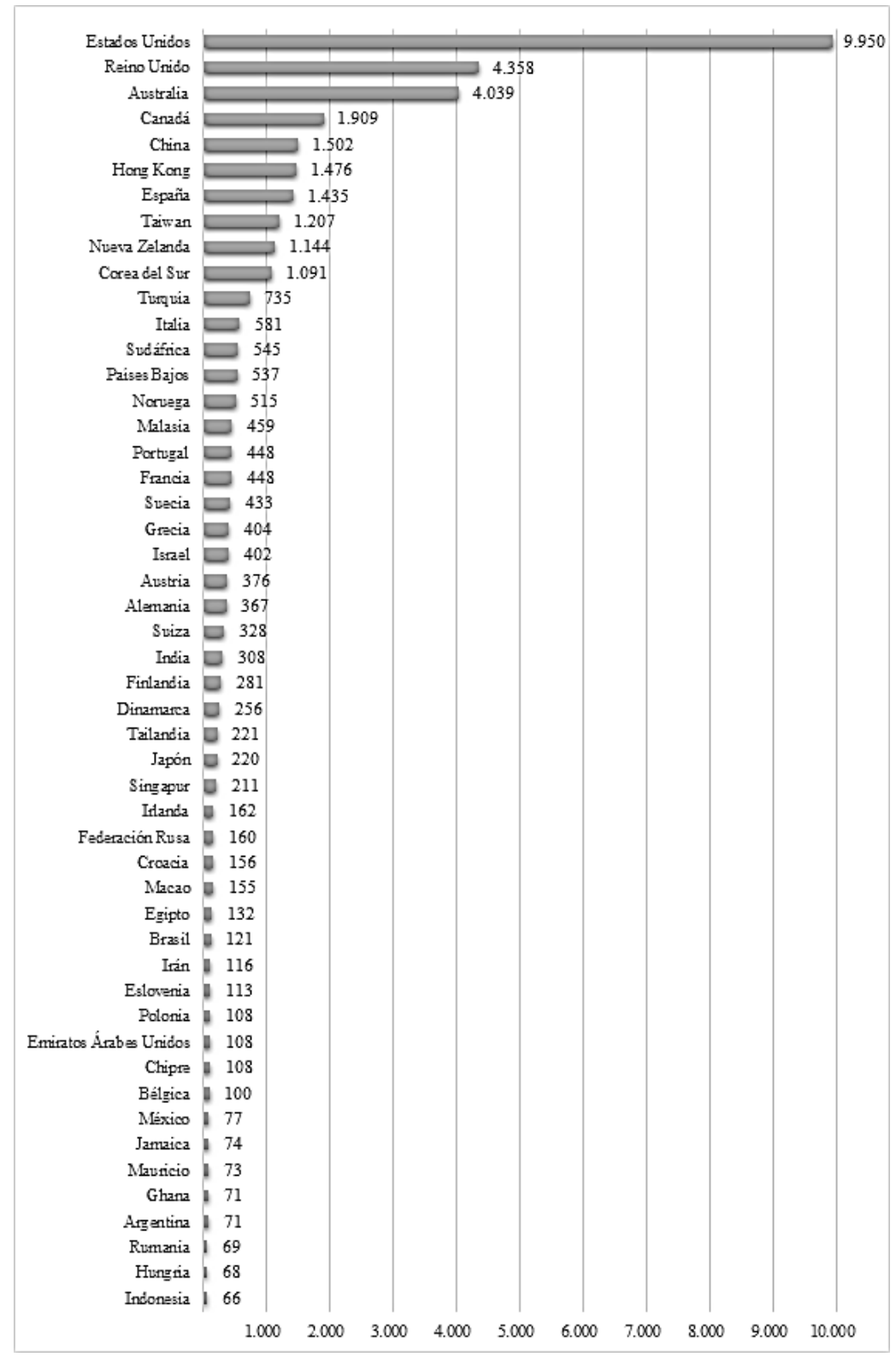

Figura 2 - Producción mundial (1998-2017).

Fuente: Scopus y elaboración propia.

Al analizar los autores más productivos, 50 publicaron 20 o más artículos y. de ellos, 27 se encuentran en centros del Reino Unido (Tabla I). Destacan Thomas Baum (University of Strathclyde) con 58 artículos y Dimitrios Buhalis (Bournemouth University) con 48 artículos, por su elevado nivel de publicación (Tabla II). El principal reto que deben afrontar los centros de investigación es atraer y 
retener a los autores altamente productivos, siendo las retribuciones y las condiciones de trabajo un elemento fundamental.

Tabla I - Producción científica, centros y autores destacados.

\begin{tabular}{|c|c|c|c|c|c|c|c|}
\hline \multirow{2}{*}{ País } & \multirow{2}{*}{$\begin{array}{c}\text { Documentos } \\
\text { 1998-2017 }\end{array}$} & \multicolumn{4}{|c|}{ Centros } & \multirow{2}{*}{$\begin{array}{l}\text { Autores } \\
\text { Líderes }\end{array}$} & \multirow{2}{*}{$\begin{array}{l}\text { Sobre } \\
\text { Mundial }\end{array}$} \\
\hline & & Top 50 & Top 100 & Top 150 & Líderes & & \\
\hline Reino Unido & 4.358 & 6 & 12 & 18 & 61 & 27 & $13,15 \%$ \\
\hline España & 1.435 & & 3 & 7 & 26 & 1 & $4,33 \%$ \\
\hline Italia & 581 & & 1 & 1 & 7 & 2 & $1,75 \%$ \\
\hline Países Bajos & 537 & & 2 & 2 & 10 & 4 & $1,62 \%$ \\
\hline Francia & 448 & & & & 4 & 2 & $1,35 \%$ \\
\hline Portugal & 448 & & 1 & 3 & 6 & 4 & $1,35 \%$ \\
\hline Suecia & 433 & & & 3 & 6 & 2 & $1,31 \%$ \\
\hline Grecia & 404 & & 1 & 1 & 8 & 1 & $1,22 \%$ \\
\hline Austria & 376 & & 1 & 2 & 6 & 3 & $1,13 \%$ \\
\hline Alemania & 367 & & & & 2 & 1 & $1,11 \%$ \\
\hline Finlandia & 281 & & 1 & 2 & 4 & 1 & $0,85 \%$ \\
\hline Dinamarca & 256 & & & 1 & 4 & 1 & $0,77 \%$ \\
\hline Irlanda & 162 & & & & 2 & & $0,49 \%$ \\
\hline Croacia & 156 & & & & 3 & & $0,47 \%$ \\
\hline Eslovenia & 113 & & & 1 & 2 & & $0,34 \%$ \\
\hline Chipre & 108 & & & & 1 & & $0,33 \%$ \\
\hline Polonia & 108 & & & & & & $0,33 \%$ \\
\hline Bélgica & 100 & & & & 1 & & $0,30 \%$ \\
\hline Rumania & 69 & & & & 1 & & $0,21 \%$ \\
\hline Hungría & 68 & & & & & & $0,21 \%$ \\
\hline Eslovaquia & 55 & & & & 1 & & $0,17 \%$ \\
\hline Bulgaria & 51 & & & & 1 & 1 & $0,15 \%$ \\
\hline Rep. Checa & 48 & & & & & & $0,14 \%$ \\
\hline Estonia & 17 & & & & & & $0,05 \%$ \\
\hline Malta & 16 & & & & & & $0,05 \%$ \\
\hline Lituania & 8 & & & & & & $0,02 \%$ \\
\hline Letonia & 5 & & & & & & $0,02 \%$ \\
\hline Luxemburgo & 3 & & & & & & $0,01 \%$ \\
\hline
\end{tabular}

Fuente: Scopus y elaboración propia.

Tabla II - Autores más productivos (1998-2017).

\begin{tabular}{|llc|}
\hline & Autor & $\begin{array}{c}\text { Documentos } \\
\mathbf{1 9 9 8 - 2 0 1 7}\end{array}$ \\
\hline Reino Unido & & \\
\hline Aberystwyth University & Garrod, B. & 21 \\
\hline Bangor University & Jones, E. & 22 \\
\hline Bournemouth University & Buhalis, D. & 48 \\
\hline
\end{tabular}




\begin{tabular}{|c|c|c|}
\hline & Ladkin, A. & 26 \\
\hline \multirow{2}{*}{ Edinburgh Napier University } & Hannam, K. & 24 \\
\hline & Leask, A. & 20 \\
\hline Global University for Lifelong Learning & Teare, $\mathrm{R}$. & 29 \\
\hline Henley Business School & Ekinci, Y. & 21 \\
\hline \multirow{2}{*}{ Leeds Beckett University } & Font, $\mathrm{X}$. & 39 \\
\hline & Thomas, R. & 23 \\
\hline Manchester Metropolitan University & Pratten, J.D. & 20 \\
\hline Oxford Brookes University & Altinay, L. & 40 \\
\hline Sheffield Hallam University & Bramwell, B. & 32 \\
\hline Swansea University & Morgan, N. & 33 \\
\hline University of Central Lancashire & Sharpley, R. & 31 \\
\hline University of Exeter & Coles, T. & 31 \\
\hline University of Hertfordshire & Page, S.J. & 37 \\
\hline University of Nottingham & McCabe, S. & 26 \\
\hline \multirow{2}{*}{ University of Strathclyde } & Baum, T. & 58 \\
\hline & Butler, R. & 34 \\
\hline \multirow{5}{*}{ University of Surrey } & Airey, D. & 38 \\
\hline & Tribe, J. & 36 \\
\hline & $\mathrm{Li}, \mathrm{G}$. & 25 \\
\hline & Riley, M. & 22 \\
\hline & Cohen, S.A. & 21 \\
\hline University of Westminster & Peng, N. & 20 \\
\hline Sin Adscripción & Pritchard, A. & 38 \\
\hline \multicolumn{3}{|l|}{ España } \\
\hline Universitat de les Illes Balears & Alegre, J. & 22 \\
\hline \multicolumn{3}{|l|}{ Italia } \\
\hline Università degli Studi di Sassari & Del Chiappa, G. & 27 \\
\hline Università Commerciale Luigi Bocconi & Baggio, R. & 22 \\
\hline \multicolumn{3}{|l|}{ Países Bajos } \\
\hline \multirow{3}{*}{ NHTV internationaal hoger onderwijs Breda } & Isaac, R.K. & 21 \\
\hline & Peeters, $\mathrm{P}$. & 21 \\
\hline & Nawijn, J. & 20 \\
\hline Tilburg University & Richards, G. & 24 \\
\hline \multicolumn{3}{|l|}{ Francia } \\
\hline \multirow{2}{*}{ Université de Bretagne Sud } & Jacob, C. & 21 \\
\hline & Guéguen, N. & 20 \\
\hline \multicolumn{3}{|l|}{ Portugal } \\
\hline Universidade do Algarve & Correia, A. & 44 \\
\hline Universidade de Lisboa & Barros, C.P. & 25 \\
\hline Universidade de Aveiro & Kastenholz, E. & 24 \\
\hline IPDT, Institute of Tourism & Costa, J. & 20 \\
\hline \multicolumn{3}{|l|}{ Suecia } \\
\hline Linnéuniversitetet & Gössling, S. & 38 \\
\hline Göteborgs universitet & Andersson, T.D. & 25 \\
\hline \multicolumn{3}{|l|}{ Grecia } \\
\hline Aristotle University of Thessaloniki & Alexandris, $\mathrm{K}$. & 21 \\
\hline \multicolumn{3}{|l|}{ Austria } \\
\hline \multirow{2}{*}{$\mathrm{MCl}$ Management Center Innsbruck } & Zehrer, A. & 39 \\
\hline & Peters, M. & 33 \\
\hline MODUL University Vienna & Smeral, E. & 27 \\
\hline
\end{tabular}




\begin{tabular}{|c|c|c|}
\hline Alemania & & \\
\hline Katholische Universität Eichstätt-Ingolstadt & Pechlaner, $\mathrm{H}$. & 27 \\
\hline \multicolumn{3}{|l|}{ Finlandia } \\
\hline Oulun Yliopisto & Saarinen, J. & 21 \\
\hline \multicolumn{3}{|l|}{ Dinamarca } \\
\hline Syddansk Universitet & Hjalager, A.M. & 24 \\
\hline \multicolumn{3}{|l|}{ Bulgaria } \\
\hline Varna University of Management & Ivanov, S. & 25 \\
\hline
\end{tabular}

Fuente: Scopus y elaboración propia.

\section{1- Evolución de los países de la UE}

La UE pasó de 197 documentos en 1998 a 1.122 en 2017, pero el porcentaje que representa sobre la producción mundial no ha variado demasiado, siendo aproximadamente el $30 \%$ a lo largo de los 20 años analizados. Por tanto, la Unión Europea ni gana ni pierde peso en el contexto mundial, a diferencia de las economías emergentes que sí ganan importancia en detrimento de los países anglosajones. Pero dentro de la UE hay dinámicas distintas según de que país se trate, aunque en mayor o menor medida casi todos están aumentando su producción científica en el campo del turismo.

Los dos países con mayor producción científica son el Reino Unido y España. Reino Unido ha duplicado su producción científica pero ya tenía elevados niveles de publicación en los años noventa. España ha tenido una mejora muy importante en los últimos cinco años y, aunque aún no lo ha conseguido, se está acercando a los niveles de Reino Unido (Figura 3).

El Reino Unido genera el $43,1 \%$ de las publicaciones de la UE, tiene 61 centros con más de 20 publicaciones (Tabla I), siendo University of Surrey el centro más productivo de la UE, y 27 autores con un elevado nivel de publicaciones (Tabla II). El peso del turismo internacional en el Reino Unido es bajo (Figura 1) pero las características adoptadas por la investigación científica desde finales de la Segunda Guerra Mundial le han beneficiado (publicaciones en inglés e importantes inversiones). Aun así, la salida del Reino Unido de la UE puede tener efectos negativos debido a la gran cantidad de fondos e investigadores que recibe del resto de la Unión y que se verá dificultada o eliminada al abandonar la UE.

España recibe elevados volúmenes de turistas internacionales desde hace décadas pero, debido al tamaño del país, la presión turística no es de las más elevadas de la UE (Figura 1). EI turismo es un sector muy importante desde los años cincuenta y, desde los noventa, se ha potenciado la formación universitaria y la investigación en turismo. En los últimos 20 años, España ha generado el $14,2 \%$ de las publicaciones académicas de la UE y tiene 26 centros con más de 20 publicaciones (Tabla I), pero sólo un investigador con más de 20 publicaciones (Tabla II). Ello indica que la investigación en turismo está muy dispersa entre muchos investigadores.

Entre los países del Mediterráneo que son miembros de la UE desde los años ochenta hay situaciones dispares. España es el que tiene la mayor producción científica, especialmente en los 
últimos cuatro años, y los otros cuatro países tienen producciones totales similares pero evoluciones distintas. Francia e Italia poseen evoluciones positivas pero sus cifras aún no se corresponden al tamaño, riqueza e importancia del turismo en esos países. Grecia era un país destacado en el campo del turismo pero, tras la crisis de 2008, su producción científica se ha estancado. Por el contrario, Portugal muestra la evolución más explosiva, especialmente en la última década. Entre los centros más destacados están Universidade do Algarve (Portugal), Universidad del Egeo (Grecia) y Libera Università di Bolzano (Italia).

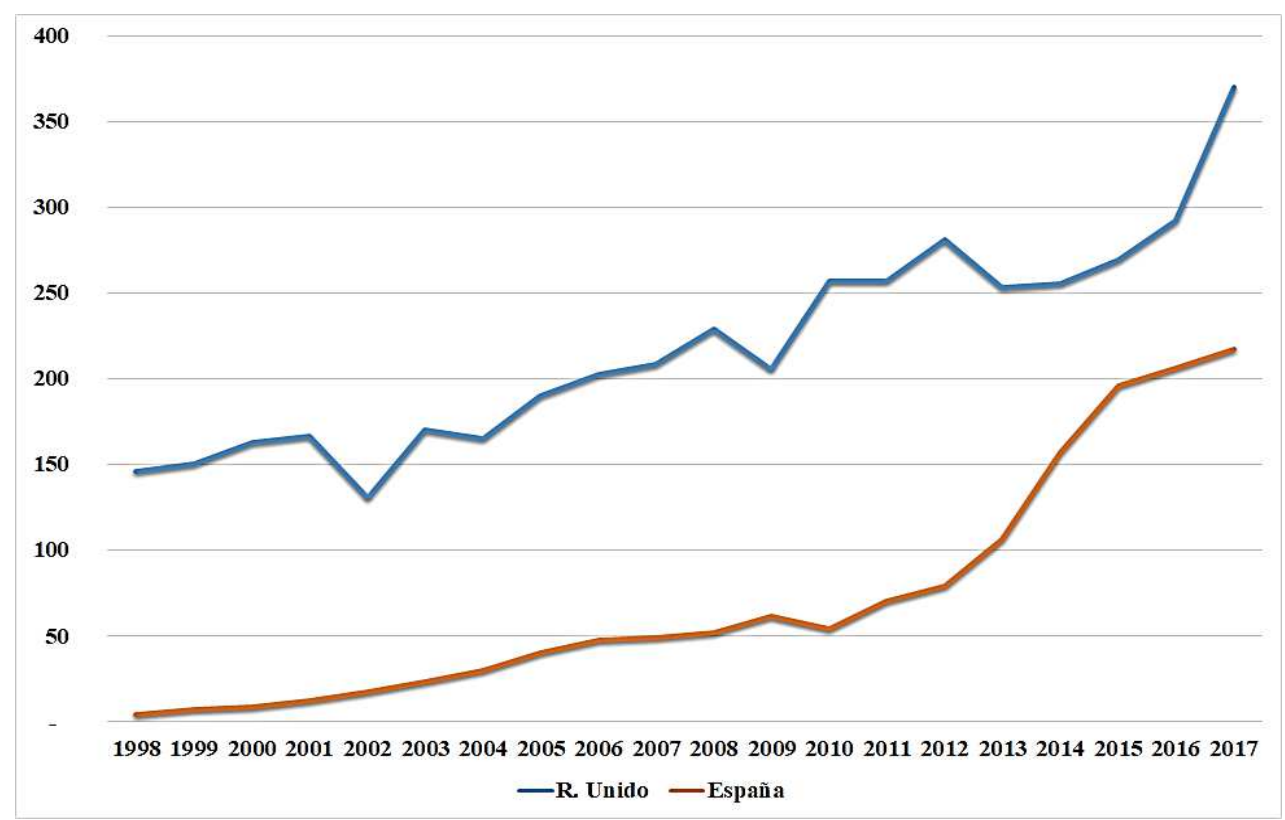

Figura 3 - Evolución de las publicaciones en Reino Unido y España. Fuente: Scopus y elaboración propia.

Los países del Mediterráneo oriental incorporados más recientemente a la UE (Chipre, Croacia y Eslovenia) son pequeños y con producciones modestas pero con una evolución positiva, salvo Chipre que tiene cierto estancamiento en los últimos años. Estos países tienen un importante desarrollo turístico (Figura 1) y realizan un gran esfuerzo en investigación, con centros que concentran gran parte de la investigación en turismo. Son los países del este de Europa que mayor esfuerzo realizan en este campo.

Alemania y Austria tienen similitudes culturales pero son puntos contrapuestos en el campo de la investigación en turismo. La producción científica de ambos países evoluciona en paralelo y con cifras similares, incluso Austria supera ligeramente a Alemania en el total de documentos (Tabla I) a pesar de tener sólo una décima parte de su población. Alemania tiene relativamente poco turismo internacional (Figura 1) y la investigación en ese campo es escasa y dispersa en múltiples centros. EI turismo no es uno de los sectores prioritarios para Alemania y, actualmente, no tiene ningún centro entre los 150 primeros del mundo (Tabla I). Austria posee una presión turística mucho más elevada (Figura 1) y, al ser un país mucho más pequeño, tiene concentrada la investigación científica en 
centros de Viena e Innsbruck. Austria es un país con una presencia importante de la actividad turística y con una gran implicación en la investigación científica.

Los países del Norte de Europa (Dinamarca, Finlandia y Suecia) tienen crecimientos lentos pero bastante constantes. Se trata de países que dedican importantes cantidades a la investigación y desarrollo, y ello tiene efecto en todos los campos de las ciencias. Cabe indicar que Noruega, que no forma parte de la UE, tiene una producción total superior a la de los otros tres países (Figura 2). Al tratarse de países pequeños, pero con significativos volúmenes de publicaciones, la investigación está bastante concentrada y hay bastantes centros con producciones elevadas: seis centros en Suecia y cuatro en Finlandia y Dinamarca. Además, los tres países tienen centros entre los 150 primeros del mundo, aunque no están posicionados muy arriba en el ranking mundial (Tabla I).

En el ámbito atlántico, además del Reino Unido, es muy importante la producción científica de Países Bajos. Países Bajos es el cuarto país del continente en publicaciones, por detrás de Italia y por delante de países como Francia y Alemania, y posee 10 centros (Tabla I) y cuatro autores (Tabla II) con más de 20 publicaciones en los últimos 20 años. De los 10 centros de investigación, dos se encuentran entre los 100 primeros del mundo, destacando NHTV internationaal hoger onderwijs Breda (puesto 61). En el campo del turismo y el ocio, el crecimiento de las publicaciones en los últimos diez años ha sido muy importante. En general, Países Bajos es un país muy competitivo en múltiples campos de investigación y ello también tiene su reflejo en turismo y ocio, a pesar de que no es uno de los países con más peso del turismo en su actividad económica (Figura 1).

Bélgica e Irlanda poseen una producción científica baja y más o menos estable, con pocos centros que destaquen y sin ningún autor con altos niveles de publicaciones (Tabla I). Tampoco son de los países con mayor desarrollo turístico (Figura 1) y, por el momento, no parece que forme parte de sus prioridades en investigación. En el caso de Irlanda, el idioma de publicación no resulta un reto, debido a que es mayoritario el uso del inglés. En Bélgica el único centro que destaca es KU Leuven, que es el centro más antiguo del país y está situado en la parte flamenca.

La mayoría de los países del este (R. Checa, Bulgaria, Eslovaquia, Hungría, Polonia, Rumania) tienen volúmenes de publicaciones pequeños. Ello implica que aún son muy escasos los centros con un volumen de publicaciones significativo (Tabla I) y ninguno está entre los 100 primeros del mundo. Estos países tienen un volumen de turistas medio o bajo (Figura 1), sin demasiada tradición en el turismo internacional, y rentas bajas. Unos bajos niveles de riqueza dificultan la generación de publicaciones de impacto internacional y obliga a priorizar inversiones. Al no ser grandes destinos turísticos, más allá de algunas ciudades como Praga, no es un sector prioritario. La evolución de los últimos cinco años hace pensar que esta situación cambiará en la próxima década y estos países se acercaran a los niveles de Europa Occidental. Portugal, Croacia y Eslovenia son posibles modelos a seguir para estos países.

Finalmente, hay cinco países con menos de 20 artículos en el periodo 1998-2017, siendo difícil un análisis detallado. Estos países son las repúblicas bálticas (Estonia, Letonia y Lituania) y los dos estados más pequeños de la UE (Luxemburgo y Malta). En el primer caso el pequeño tamaño y los 
bajos niveles de renta explican sus bajas cifras. En el segundo caso, el pequeño tamaño es la principal limitación. Hasta el momento, ninguno de estos países ha generado más de cuatro artículos en un mismo año pero es posible que aumente el número de publicaciones en el futuro, dada la tendencia mundial y europea.

\section{2- Comparativa de los países de la UE}

Si no se tienen en cuenta las diferencias entre países (Figura 4), los más importantes son Reino Unido y España, seguidos por Italia y Países Bajos. Pero los países que conforman la UE tienen importantes diferencias en superficie, población, desarrollo turístico y renta, debiendo relativizar la producción científica. EI PIB total de un país en Paridad de Poder de Compra puede ser un buen indicador del tamaño y riqueza. Al relativizar la producción científica (Figura 5), Chipre es el país que obtiene mejores resultados, seguido de Reino Unido, Eslovenia y Croacia. Por el contrario, los países que conforman el centro geográfico de la UE (Francia, Bélgica, Alemania, Polonia, etc.) tienen los niveles más bajos de producción científica en turismo.

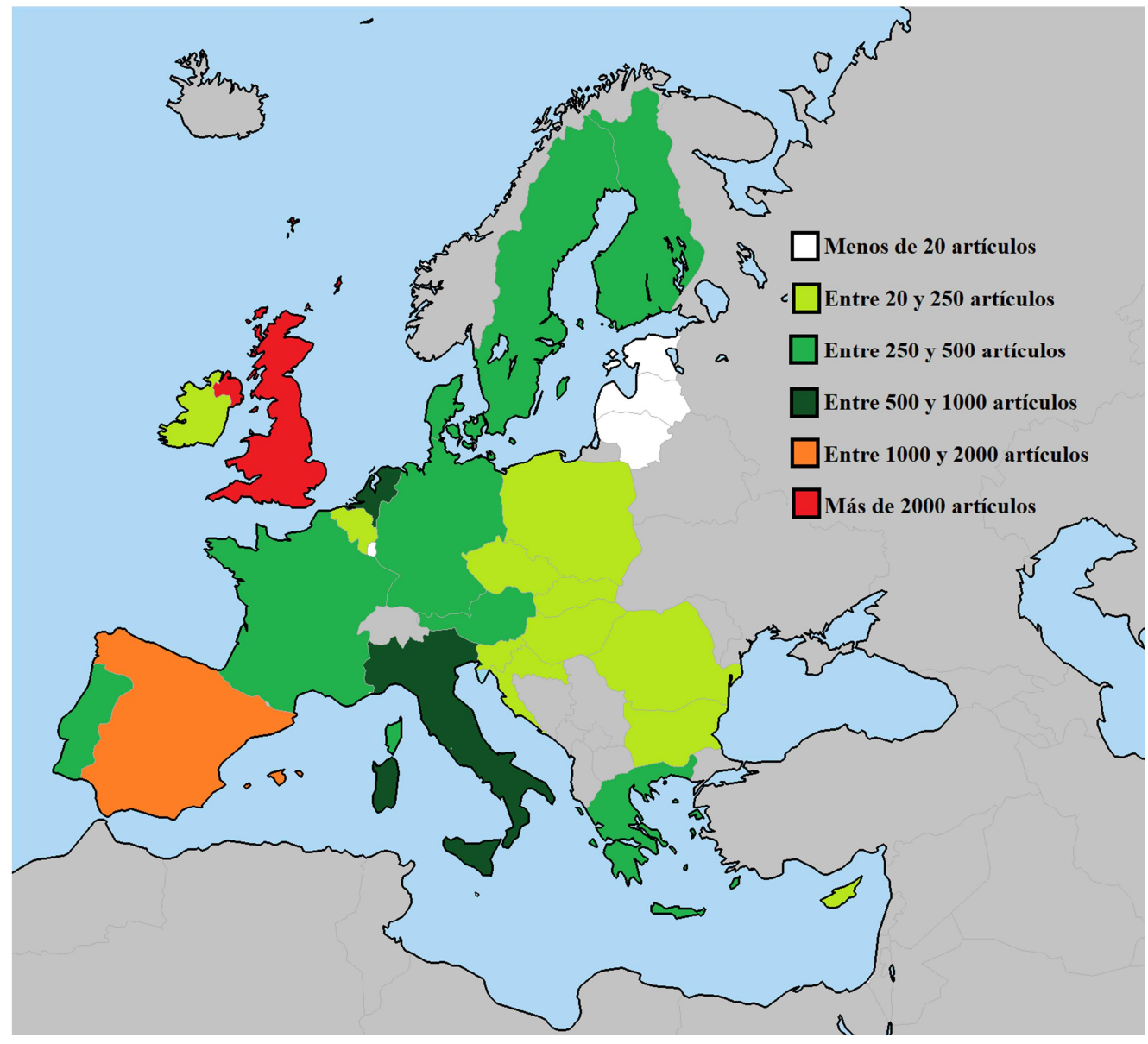

Figura 4 - Producción total (1998-2017).

Fuente: Scopus y elaboración propia. 


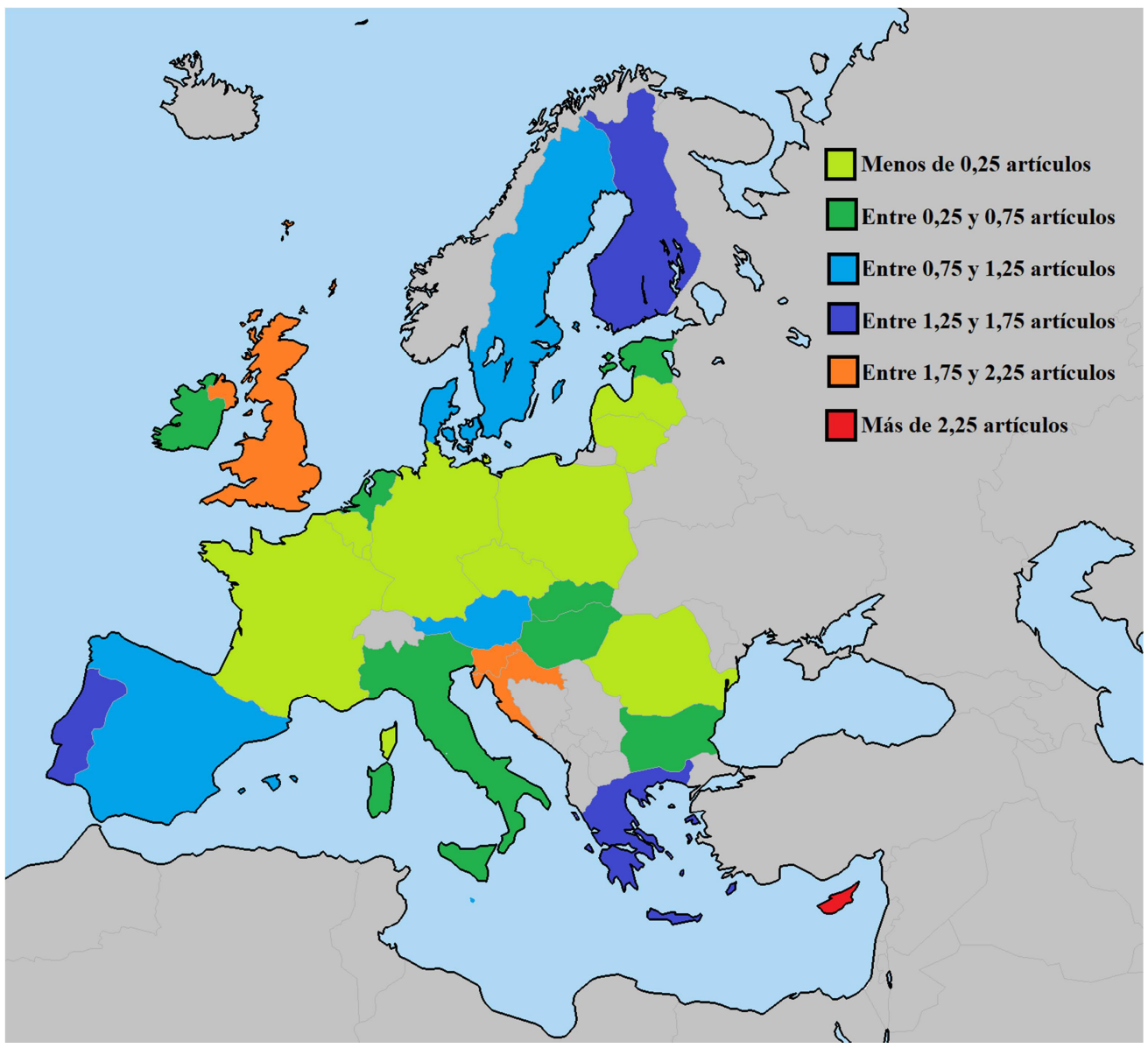

Figura 5 - Producción relativa (1998-2017) comparado con PIB 2017 (1.000 mill. €). Fuente: Central Intelligence Agency, UNWTO (2017), Scopus y elaboración propia.

\section{Conclusiones}

Al tener en cuenta el tamaño del país y su riqueza (medida con el PIB), destacan dos tipos de países por su producción científica: los países del Mediterráneo, excepto Italia y Francia; y un grupo de países con alta implicación en investigación (Reino Unido, Finlandia, Dinamarca, Suecia y Austria). Entre los países con una producción baja hay dos grupos: diversos países centrales con poca especialización en turismo (Luxemburgo, Alemania, Francia, Bélgica e Italia); y los países del este (Lituania, Polonia, Letonia, Republica Checa, Rumanía, Hungría, etc.).

Los países del este poseen niveles de riqueza inferiores al resto y eso dificulta la generación de publicaciones en revistas de alto impacto, más aún en sectores con poca tradición como sector económico, como es el caso del turismo. En este contexto hay que destacar el esfuerzo de Croacia y Eslovenia, especialmente el de algunas de sus universidades. En Europa occidental hay que mencionar el caso de Portugal, ya que ha conseguido mejorar significativamente su producción científica durante la última década, a pesar de la situación económica del país. Ello se ha debido al esfuerzo de los investigadores portugueses por captar fondos europeos que suplan la falta de 
financiación nacional. La situación contraria a la de Portugal es la de Grecia, al vivir un estancamiento en la última década que le ha hecho perder peso internacional en el campo del turismo.

Aunque la producción científica de la UE ha aumentado, gracias a que cada vez más centros y países realizan investigación en este campo, sólo representó el 33,7\% del total mundial en 2016, no mucho más del $29,9 \%$ de 1998 . Ello es debido al rápido crecimiento de las publicaciones sobre turismo en el mundo, principalmente en las economías emergentes. Aun así es una evolución mejor que la de los países anglosajones, que han crecido menos que el total mundial y pierden peso relativo. Ese 33,7\% mundial de 2016 es inferior al volumen de turistas internacionales recibidos ese año $(40,5 \%)$. Ello indica que la investigación académica europea aún no se encuentra al nivel de su sector económico.

La salida de Reino Unido puede mermar significativamente los resultados de la UE y del propio Reino Unido. El Reino Unido ha participado en el $43,1 \%$ de las publicaciones en turismo de la UE, muchas de ellas colaboraciones entre investigadores británicos y de otros países europeos. Sin el Reino Unido, las publicaciones totales de la UE se verán reducidas significativamente, pero se mantendrán colaboraciones y parte de la actividad científica del Reino Unido se trasladará a centros del continente.

Aunque hay importantes crecimientos en los volúmenes de publicaciones, diversos países cuentan con cifras muy bajas debido a que este tipo de publicaciones tienen dificultades que perjudican a los países más pequeños y pobres:

- El acceso a la bibliografía internacional es de pago en la mayoría de casos. El coste del acceso a estas publicaciones es muy elevado, incluso para los centros punteros de los países más ricos, y prohibitivo para los países pobres. Para contrarrestar esta barrera se han creado acumuladores online. Se trata de un caso de "piratería" que busca superar la barrera de los elevados costes de acceso a las publicaciones de las grandes editoriales.

- La redacción de los trabajos académicos implica dificultades, no sólo por el aprendizaje de los estilos típicos en los trabajos académicos. El predominio del inglés en las revistas académicas internacionales deja en desventaja a los autores no anglosajones, ya que deben exponer cuestiones complejas en un idioma que no es el suyo. Ello implica costes en aprendizaje del inglés, y en traducción y revisión de textos. También es motivo de muchas de las colaboraciones con investigadores anglosajones.

Finalmente, cabe indicar que la investigación en turismo es muy reciente y vive una expansión exponencial que hace que en cinco o diez años la situación internacional cambie significativamente. Los análisis bibliométricos deben realizarse periódicamente y para todos los países con la finalidad de detectar cambios de tendencia o nuevos centros y autores con potencial internacional.

La principal limitación de este trabajo está en que no se han ponderado las diversas publicaciones tomadas en consideración y no se han tenido en cuenta otras revistas especializadas de menor relevancia. Otra limitación es el funcionamiento del buscador de la base Scopus, ya que muestra algunas imperfecciones que hacen difícil repetir los mismos resultados con total exactitud. 


\section{Bibliografía}

BALLANTYNE, R., PACKER, J., \& AXELSEN, M. (2009). Trends in tourism research. Annals of Tourism Research, 36(1), 149-152.

CENTRAL INTELLIGENCE AGENCY (CIA). The World Factbook. Washington, D.C.: Central Intelligence Agency. Disponible en: https://www.cia.gov/library/publications/the-world-factbook/ [acceso el 15 de marzo de 2018]

CHANG, C. L., \& MCALEER, M. (2012). Citations and impact of ISI tourism and hospitality journals. Tourism Management Perspectives, 1, 2-8.

FERREIRA, R. R., DEFRANCO, A. L., \& RAPPOLE, C. L. (1994). Rating the hospitality journals. International Journal of Hospitality Management, 13(3), 209-218.

FRECHTLING, D. C. (2004). Assessment of tourism/hospitality journals' role in knowledge transfer: an exploratory study. Journal of Travel Research, 43(2), 100-107.

HALL, C. M. (2011). Publish and perish? Bibliometric analysis, journal ranking and the assessment of research quality in tourism. Tourism Management, 32(1), 16-27.

JAMAL, T., SMITH, B., \& WATSON, E. (2008). Ranking, rating and scoring of tourism journals: Interdisciplinary challenges and innovations. Tourism Management, 29(1), 66-78.

JOGARATNAM, G., CHON, K., MCCLEARY, K., MENA, M., \& YOO, J. (2005). An analysis of institutional contributors to three major academic tourism journals: 1992-2001. Tourism Management, 26(5), 641-648.

KIM, Y., SAVAGE, K. S., HOWEY, R. M., \& VAN HOOF, H. B. (2009). Academic foundations for hospitality and tourism research: a reexamination of citations. Tourism Management, 30(5), $752-758$.

LI, J., \& XU, Y. (2015). Author analyses of tourism research in the past thirty years-Based on ATR, JTR and TM. Tourism Management Perspectives, 13, 1-6.

MCKERCHER, B. (2005). A case for ranking tourism journals. Tourism Management, 26(5), 649-651.

MCKERCHER, B. (2008). A citation analysis of tourism scholars. Tourism Management, 29(6), 12261232.

MCKERCHER, B., LAW, R., \& LAM, T. (2006). Rating tourism and hospitality journals. Tourism Management, 27(6), 1235-1252.

PARK, K., PHILLIPS, W. J., CANTER, D. D., \& ABBOTT, J. (2011). Hospitality and tourism research rankings by author, university, and country using six major journals: The first decade of the new millennium. Journal of Hospitality \& Tourism Research, 35(3), 381-416.

PECHLANER, H., ZEHRER, A., MATZLER, K., \& ABFALTER, D. (2004). A ranking of international tourism and hospitality journals. Journal of Travel Research, 42(4), 328-332.

RAMÓN, J. (2017). Reflexiones sobre el turismo en el ámbito académico español. REDMARKA: Revista Digital de Marketing Aplicado, 18(1), 83-121.

RYAN, C. (2005). The ranking and rating of academics and journals in tourism research. Tourism Management, 26(5), 657-662.

SCOPUS. Disponible en: https://www.scopus.com/ [acceso el 10 de febrero de 2018]

SEVERT, D. E., TESONE, D. V., BOTTORFF, T. J., \& CARPENTER, M. L. (2009). A world ranking of the top 100 hospitality and tourism programs. Journal of Hospitality \& Tourism Research, 33(4), 451-470.

SHELDON, P. J. (1991). An authorship analysis of tourism research. Annals of Tourism Research, 18(3), 473-484. 
SVENSSON, G., SVAERI, S., \& EINARSEN, K. (2009). "Empirical characteristics" of scholarly journals in hospitality and tourism research: an assessment. International Journal of Hospitality Management, 28(3), 479-483.

UNWTO (2017). Panorama OMT del turismo internacional: Edición 2017. Madrid: UNWTO.

VARGAS, A. (2011). ¿Los principales destinos son también las principales potencias en la investigación en turismo? Estudios Turísticos, 188, 91-111.

ZHAO, W., \& RITCHIE, J. R. B. (2007). An investigation of academic leadership in tourism research: 1985-2004. Tourism Management, 28(2), 476-490. 